\title{
DEFORMATIONS OF EXCEPTIONAL WEIERSTRASS POINTS
}

\author{
STEVEN DIAZ
}

\begin{abstract}
Using first order deformation theory of pointed curves we show that the semigroup of a generic Weierstrass point whose semigroup has first nonzero element $k$ consists only of multiples of $k$ until after its greatest gap value, and that on the moduli space of curves two components of the divisor of points corresponding to curves possessing exceptional Weierstrass points intersect nontransversely.
\end{abstract}

As usual let $\mathscr{M}_{g}$ be the moduli space of curves of genus $g$. Many subloci of $\mathscr{M}_{g}$ defined in terms of Weierstrass points have received much study.

(1) Definition. For an integer $k$ with $2 \leqslant k \leqslant g, D_{k, k}=\left\{[C] \in \mathscr{M}_{g}: C\right.$ possesses a Weierstrass point $p$ with $\left.h^{0}(C, k p) \geqslant 2\right\}$.

It is known that $D_{k, k}$ is irreducible of dimension $2 g-3+k$ and a general point in $D_{k, k}$ corresponds to a curve $C$ with a Weierstrass point $p$ with $h^{0}(C, k p)=2$. See Rauch [1], Lax [1, 2], Arbarello [1, 2]. Here we will prove:

(2) THEOREM. A generic point in $D_{k, k}$ corresponds to a curve with a Weierstrass point whose semigroup consists only of multiples of $k$ until after the greatest gap.

(For $k \geqslant \frac{1}{2} g+1$ this also follows from results in Eisenbud-Harris [1].)

There are other subsets of $\mathscr{M}_{g}$ defined in terms of Weierstrass points.

(3) Definition. For an integer $k$ with $0 \leqslant k \leqslant g-2, E(k)=\left\{[C] \in \mathscr{M}_{g}: C\right.$ possesses a Weierstrass point $p$ with $\left.h^{0}(C,(g+k) p) \geqslant k+2\right\}$. It is known that $E(k)$ has dimension $3 g-3-k$. See for instance Diaz [1]. By carrying the calculations used to prove (2) a little further we will demonstrate some nontransversality between the subvarieties $D_{k, k}$ and $E(j)$.

(4) TheOREM. At a point $[C] \in \mathscr{M}_{g}$ corresponding to a curve $C$ of genus $g \geqslant 4$ with $a$ Weierstrass point $p$ with gap sequence $1,2, \ldots, g-2, g, g+2$ the branches of $D_{g-1, g-1}$ and $E(1)$ corresponding to $p$ have the same tangent space. Thus the intersection of the two divisors $D_{g-1, g-1}$ and $E(1)$ has at least one multiple component.

We work over the complex numbers.

Here is the strategy of the proof of (2). It proceeds by contradiction. Assume that on a generic curve $C$ corresponding to a point in $D_{k, k}$ the indicated Weierstrass point $p$ has a semigroup containing numbers less than the largest gap which are not multiples of $k$. We show that under a general deformation of $C$ remaining in $D_{k, k}$ the functions which gave rise to these numbers cannot also deform with their poles

Received by the editors August 22, 1984.

1980 Mathematics Subject Classification. Primary 14F07; Secondary 14B12, 14H10. 
concentrated on the deformation of $p$. This is done using a theory of first order deformations of pointed curves developed in Diaz [1]. First we summarize some necessary results.

Consider a smooth curve $C$ and $n$ marked points $p_{1}, \ldots, p_{n}$ on $C$. Let $\theta$ be the tangent bundle, $K$ the canonical bundle, and $\mathcal{O}$ the structure sheaf of $C$. First order deformations of $C$ are classified by $H^{1}(C, \theta)$ and first order deformations of $C$ together with $p_{1}, \ldots, p_{n}$ are classified by $H^{1}\left(C, \theta\left(-p_{1}-\cdots-p_{n}\right)\right)$. Given a rational function $f$ on $C$ whose divisor is supported set theoretically on $p_{1}, \ldots, p_{n}$ and an element $\phi$ of $H^{1}\left(C, \theta\left(-p_{1}-\cdots-p_{n}\right)\right)$, then it is possible to deform $f$ along the deformation of $C, p_{1}, \ldots, p_{n}$ given by $\phi$ so that the divisor of $f$ remains supported on the deformations of the points if and only if the cup product $\phi \cdot \mathrm{d} \log f=0$ in $H^{1}(C, \mathcal{O})$.

One is usually interested in sets of the form $\left\{\phi \in H^{1}\left(C, \theta\left(-p_{1}-\cdots-p_{n}\right)\right)\right.$ : $\phi \cdot \mathrm{d} \log f=0\}$. By Kodaira-Serre duality this is the same as the annihilator of the image of the multiplication map.

$$
H^{0}(C, K) \otimes\{\mathrm{d} \log f\} \rightarrow H^{0}\left(C, 2 K\left(p_{1}+\cdots+p_{n}\right)\right) .
$$

Proof of (2). The theorem is well known when $k=2$ or $g$ so let us assume $2<k<g$, which also means $g>3$. Let $C$ be a curve corresponding to a generic point of $D_{k, k}$. By Diaz [1, Theorem 4.9] we may take $p$ to be the unique point on $C$ with $h^{0}(C, k p)=2$. Assume there is an integer $l$ in the semigroup of $p$ which is not a multiple of $k$ and is less than the greatest gap. We may take $l$ to be the smallest such integer.

There are rational functions $f_{1}$ and $f_{2}$ on $C$ with $\left(f_{1}\right)=-k p+q(1,1)$ $+\cdots+q(1, k)$ and $\left(f_{2}\right)=-l p+q(2,1)+\cdots+q(2, l)$. (In what follows the questions are local so if $C$ has automorphisms one may work in the universal deformation space of $C$, instead of $\mathscr{M}_{g}$.)

Near $[C], D_{k, k}$ is the subvariety of $\mathscr{M}_{g}$ corresponding to deformations of $C$ for which it is also possible to deform $p, q(1,1), \ldots, q(1, k)$ and $f_{1}$ so that the divisor of $f_{1}$ remains supported on the deformations of the points. In $\mathscr{C}_{g}$, the universal curve, define $V D_{k, k}$ to be the locus near $p$ of Weierstrass points $s$ such that if $C_{s}$ is the curve on which $s$ lies, $h^{0}\left(C_{s}, k s\right)=2$. Similarly, near $[C]$ define $W(k, l)$ to be the subvariety of $\mathscr{M}_{g}$ corresponding to deformations of $C$ for which it is also possible to deform $f_{1}, f_{2}, p, q(1,1), \ldots, q(1, k), q(2,1), \ldots, q(2, l)$ so that the divisors of $f_{1}$ and $f_{2}$ stay on the deformations of the appropriate points. Define $V W(k, l)$ to be the subvariety of $\mathscr{C}_{g}$ near $p$ of corresponding Weierstrass points. To prove the theorem we must show that $V D_{k, k} \neq V W(k, l)$.

Using the previously mentioned deformation theory one calculates the tangent spaces to these subvarieties at $[C]$ or $p$.

$T D_{k, k}=$ annihilator of $H^{0}(C, K-p-q(1,1)-\cdots-q(1, k)) \otimes \mathrm{d} \log f_{1}$,

$T V D_{k, k}=$ annihilator of $H^{0}(C, K-q(1,1)-\cdots-q(1, k)) \otimes \mathrm{d} \log f_{1}$,

$T W(k, l)=$ annihilator of all regular quadratic differentials in

$$
H^{0}(C, K) \otimes \operatorname{span}\left\{\mathrm{d} \log f_{1}, \mathrm{~d} \log f_{2}\right\},
$$


$T V W(k, l)=$ annihilator of all rational quadratic differentials whose only pole

if any is a simple pole at $p$ in $H^{0}(C, K) \otimes \operatorname{span}\left\{\mathrm{d} \log f_{1}, \mathrm{~d} \log f_{2}\right\}$.

Clearly $h^{0}(C, K-q(1,1)-\cdots-q(1, k))=h^{0}(C, K-k p)=g-k+1$. In Diaz [1, Theorem 4.8] it was shown that $h^{0}(C, K-p-q(1,1)-\cdots-q(1, k))=g$ $-k$. This shows that the dimensions of the tangent spaces to $D_{k, k}$ and $V D_{k, k}$ are equal to the dimensions of these varieties. Therefore to prove (2) we may show either $T D_{k, k} \not \subset T W(k, l)$ or $T V D_{k, k} \not \subset T V W(k, l)$.

Case 1. $l \leqslant g$. Assume that $T D_{k, k} \subset T W(k, l)$. Since $h^{0}(C, l p) \geqslant 3$, we know that $h^{0}(C, K-p-q(2,1)-\cdots-q(2, l)) \geqslant 1$. So there exists a regular differential $\phi$ such that $\phi \mathrm{d} \log f_{2}$ is regular. Due to the assumed containment of tangent spaces this means there must be a regular differential $\omega$ such that

$$
\omega \mathrm{d} \log f_{1}=\phi \mathrm{d} \log f_{2} \text {. }
$$

Let $B_{i}$ be the branch divisor of $f_{i}$ away from $p$. Counting zeros and poles in (7) one concludes that $B_{1} \cap B_{2}$ is nonempty.

Case 1a. $B_{1} \not \subset B_{2}$. The choice of $f_{1}$ is unique up to scalars. The choice of $f_{2}$ is unique only up to powers of $f_{1}$ with poles of order less than $l$ at $p$. One easily checks that these possible changes do not affect $B_{1} \cap B_{2}$. This means that $B_{1}$ has a marked subset, its intersection with $B_{2}$. This contradicts Diaz [1, Lemma 3.2] where it was shown that the moduli space of $\left\{k\right.$ sheeted covers of $P^{1}$ of genus $g$ with one point of total ramification and the rest of the ramification simple and over distinct points plus an ordering of the simple branch points $\}$ is connected.

Case 1b. $B_{1} \subset B_{2}$. Consider $\left(d f_{2}\right)\left(d f_{1}\right)^{-1}$. It is a rational function whose only pole is one of order $l-k$ at $p$ contradicting our choice of $l$ and $k$.

Case 2. $l>g$. Assume $T V D_{k, k} \subset T V W(k, l)$. Since

$$
h^{0}(C, K-q(2,1)-\cdots-q(2, l)) \geqslant 1,
$$

the containment of tangent spaces still implies a relation as in (7), except now the quadratic differentials may have simple poles at $p$. The proof then proceeds as in Case 1.

This completes the proof of (2). We now show some cases where $T D_{k, k}=T W(k, l)$. Retain the same notation as in the proof. If $l$ is exactly one less than the last gap and there are $j$ positive multiples of $k$ less than $l$, then $[C]$ is a point in $E(j)$. Near $[C]$, $W(k, l)$ will be a component of $D_{k, k} \cap E(j)$. (We do not know whether $C$ may have other exceptional Weierstrass points.) The tangent space to the branch of $E(j)$ corresponding to $p$ can be calculated using the previously mentioned deformation theory. This was done in Diaz [1, Theorem 4.13].

(8) Let $\omega$ be the unique regular differential vanishing to order $l=g+j$ at $p$. Set $\omega_{i}=f_{1}^{i} \omega, i=1, \ldots, j$. Then the tangent space to the branch of $E(j)$ corresponding to $p$ at $[C]$ is the annihilator of the span of $\left\{\omega_{1} \mathrm{~d} \log f_{1}, \ldots, \omega_{j} \mathrm{~d} \log f_{1}\right\}$.

But clearly $\omega_{1}, \ldots, \omega_{j}$ are linearly independent elements of

$$
H^{0}(C, K-p-q(1,1)-\cdots-q(1, k)) \text {. }
$$

Therefore at $[C]$ comparing (8) with (6) we see that, for the branches corresponding to $p, T D_{k, k} \subset T E(j)$, so $T D_{k, k}=T W(k, l)$. 
In general, it is not known whether Weierstrass points with gap sequences of the type we have been discussing since the end of the proof of (2) exist. If $k \geqslant \frac{1}{2} g+1$ Eisenbud-Harris [1] shows that such Weierstrass points do exist. In fact when the gap sequence is $1,2, \ldots, g-2, g, g+2$ they show that the set of all points in $\mathscr{M}_{g}$ corresponding to curves with such Weierstrass points has at least one component of codimension 2. This completes the proof of (4).

Arbarello, E.

REFERENCES

1. On subvarieties of the moduli space of curves of genus $g$ defined in terms of Weierstrass points, Atti Accad. Naz. Lincei Mem. Cl. Sci. Fis. Mat. Natur. Sez. Ia (8) 15 (1978), 3-20.

2. Weierstrass points and moduli of curves, Compositio Math. 29 (1974), 325-342. Diaz, $\mathbf{S}$.

1. Tangent spaces in moduli via deformations with applications to Weierstrass points, Duke Math. J. (to appear).

Eisenbud D. and J. Harris

1. Limit linear series, the irrationality of $\mathscr{M}_{g}$, and other applications, Bull. Amer. Math. Soc. (N.S.) 10 (1984), 277.

Lax, R.F.

1. On the dimension of varieties of special divisors, Trans. Amer. Math. Soc. 203 (1975), 141-159.

2. Weierstrass points on the universal curve, Math. Ann. 216 (1975), 35-42.

Rauch, $\mathrm{H}$.

1. Weierstrass points, branch points, and moduli of Riemann surfaces, Comm. Pure Appl. Math. 12 (1959), 543-560.

Department of Mathematics, University of Pennsylvania, Philadelphia, Pennsylvania 19104

Current address: Department of Mathematics, Brandeis University, Waltham, Massachusetts 02254 